\title{
New Global Synchronization Analysis for Complex Networks with Coupling Delay
}

\author{
Jinfang Zhang, ${ }^{1}$ Yuanhua Qiao, ${ }^{1}$ Jun Miao, ${ }^{2}$ Lijuan Duan, ${ }^{3}$ and Yanjun Zeng ${ }^{4}$ \\ ${ }^{1}$ College of Applied Sciences, Beijing University of Technology, Beijing 100124, China \\ ${ }^{2}$ Key Laboratory of Intelligent Information Processing, Institute of Computing Technology, Chinese Academy of Sciences, \\ Beijing 100190, China \\ ${ }^{3}$ College of Computer Science, Beijing University of Technology, Beijing 100124, China \\ ${ }^{4}$ College of Life Science and Bioengineering, Beijing University of Technology, Beijing 100124, China
}

Correspondence should be addressed to Yuanhua Qiao; qiaoyuanhua@bjut.edu.cn and Jun Miao; jmiao@ict.ac.cn

Received 27 October 2013; Accepted 21 December 2013; Published 12 March 2014

Academic Editor: Qiankun Song

Copyright (C) 2014 Jinfang Zhang et al. This is an open access article distributed under the Creative Commons Attribution License, which permits unrestricted use, distribution, and reproduction in any medium, provided the original work is properly cited.

Global synchronization analysis for complex networks with coupling delay is investigated. Firstly the constant time delay is analyzed and then the case for time-varying delay is considered. Sufficient conditions for network synchronization are given based on Lyapunov functional, linear matrix inequality, and Kronecker product technique. The unknown variables in the sufficient conditions are fewer than those in the recent reference. Moreover, for the time-varying delay case, we find that the conditions are dependent on the bounds of both time delay and its derivative, and the derivative of the time-varying delay can be any value in the bounds. Finally, numerical examples are given to validate the effectiveness of the obtained results.

\section{Introduction}

Dynamical recurrent neural networks are used extensively in classification of patterns, associative memories, optimization [1-3], and so on. The networks are composed of a large number of highly interconnected dynamical units and exhibit very complicated dynamics. Therefore, theory analysis of complex networks has become a focal research field and attracted a great deal of attention.

Recently, it is found that synchronization is one of the most important dynamical properties of complex networks and has been extensively investigated in different ways [419]. In [5], Lü and Chen considered a dynamical network and gave the sufficient conditions for local synchronization. Because of the network traffic congestions as well as the finite speed of signal transmission over the links, time delays occur commonly in complex networks. Therefore, Zhou and Chen [7] and Gao et al. [8] improved the models with no delays, the case with constant coupling delays is considered, and they also analyzed the synchronization problem $[9,10]$. However, in many real-world networks time delay is varying; therefore time varying coupling delays are considered in [11-13]. By using free-weighting matrices, some synchronization criteria for general complex dynamical networks with time-varying delays are proposed. However, the computation is huge, and there are a large amount of variables in the condition. Moreover, in order to derive the synchronization condition, in some papers [7-13], the time-varying delay $\tau(t)$ is usually confined to $0 \leq \tau(t) \leq \tau$ (lower bound of the delay is zero), and the derivative is restricted to less than 1 . Therefore, how to improve the system performance by removing the redundant variables and reducing computation still remains unsolved.

Motivated by the mentioned work, we study the synchronization problems for general complex networks with time constant coupling delays and interval time-varying delays. Using different Lyapunov functions, the synchronization conditions derived turn out to be less conservative, and the addressed systems contain some models as their special cases; the more effective mathematical techniques are employed to reduce the conservatism.

The remainder of this paper is organized as follows. In Section 2, the investigated systems are formulated and some lemmas and notations are given. In Section 3, the conditions for synchronization are derived. In Section 4, two numerical 
examples are presented to demonstrate the effectiveness and the advantage of the proposed method. Finally, conclusions are drawn in Section 5.

\section{Problem Formulations}

In this paper, $R^{m \times n}$ denotes the set of $m \times n$ real matrixes, and $X \geq 0$ denotes that matrix $X$ is positive semidefinite, while $X>0$ denotes that matrix $X$ is positive definite. We use $\operatorname{diag}\{\cdots\}$ to denote a block-diagonal matrix; $\left[\begin{array}{ll}X & Y \\ * & Z\end{array}\right]$ stands for $\left[\begin{array}{cc}X & Y \\ Y^{T} & Z\end{array}\right]$. The notation $A \otimes B$ denotes the Kronecker product of matrices $A$ and $B ; I_{n}$ represents the $n$-dimensional identity matrix. Matrix dimensions, if not explicitly stated, are assumed to be compatible for algebraic operations.

We use $x_{i}(t)$ to denote the state of coupling nodes, $i \in$ $\{1, \ldots, N\}$, and then the dynamic neural networks (DNNs) of general form can be described by

$$
\begin{aligned}
\dot{x}_{i}(t)= & -C x_{i}(t)+D f\left(x_{i}(t)\right)+B f\left(x_{i}(t-\tau)\right)+I(t) \\
& +c \sum_{j=1, j \neq i}^{N} G_{i j} A\left[x_{j}(t-\tau)-x_{i}(t-\tau)\right] \\
& +c \sum_{j=1, j \neq i}^{N} G_{i j} A_{\tau}\left[x_{j}(t-\tau)-x_{i}(t)\right], \\
\dot{x}_{i}(t)= & -C x_{i}(t)+D f\left(x_{i}(t)\right)+B f\left(x_{i}(t-\tau(t))\right)+I(t) \\
& +c \sum_{j=1, j \neq i}^{N} G_{i j} A\left[x_{j}(t-\tau(t))-x_{i}(t-\tau(t))\right] \\
& +c \sum_{j=1, j \neq i}^{N} G_{i j} A_{\tau}\left[x_{j}(t-\tau(t))-x_{i}(t)\right],
\end{aligned}
$$

in which $x_{i}(t)=\left[x_{i 1}(t), x_{i 2}(t), \ldots, x_{i_{n}}(t)\right]^{T} \in R^{n}$ is the state vector of the $i$ th network at time $t$. The functions $f\left(x_{i}(t)\right)=\left[f_{1}\left(x_{i 1}(t)\right), f_{2}\left(x_{i 2}(t)\right), \ldots, f_{n}\left(x_{i n}(t)\right)\right]^{T}$ are sufficiently smooth nonlinear vector fields, and $I(t)=\left[I_{1}(t)\right.$, $\left.I_{2}(t), \ldots, I_{n}(t)\right]^{T} \in R^{n}$ is the external input vector. $C=\left[c_{i j}\right]_{n \times n}$, $D=\left[d_{i j}\right]_{n \times n}$, and $B=\left[b_{i j}\right]_{n \times n}$ are coefficient matrixes, $A=$ $\left[a_{i j}\right]_{n \times n}$ and $A_{\tau}=\left[a_{i j}^{\prime}\right]_{n \times n}$ are inner-coupling matrixes, and $G=\left[G_{i j}\right]_{N \times N}$ represents the outer-coupling connections. The constant $c>0$ represents the coupling strength; $\tau$ and $\tau(t)$ represent the time constant delay and time-varying delay, respectively.

For the networks (1), we have the following assumptions.

Assumption (H1). $\tau(t)$ is the interval time-varying delay satisfying

$$
\begin{gathered}
0 \leq h_{1} \leq \tau(t) \leq h_{2}, \\
\dot{\tau}(t) \leq \mu<+\infty, \\
h_{21}=h_{2}-h_{1} .
\end{gathered}
$$

Assumption (H2). The outer-coupling configuration matrices of the network satisfy

$$
\begin{gathered}
G_{i j} \geq 0, \quad i \neq j, \\
G_{i i}=-\sum_{j=1, j \neq i}^{N} G_{i j}, \quad i, j=1,2, \ldots, N .
\end{gathered}
$$

Assumption (H3). There exist constants $\sigma_{i}^{-}, \sigma_{i}^{+}$, and the functions satisfy

$$
\sigma_{i}^{-} \leq \frac{f_{i}\left(x_{1}\right)-f_{i}\left(x_{2}\right)}{x_{1}-x_{2}} \leq \sigma_{i}^{+}, \quad i=1,2, \ldots, n .
$$

We denote

$$
\begin{gathered}
\Lambda_{1}=\operatorname{diag}\left(\sigma_{1}^{+} \sigma_{1}^{-}, \sigma_{2}^{+} \sigma_{2}^{-}, \ldots, \sigma_{n}^{+} \sigma_{n}^{-}\right), \\
\Lambda_{2}=\operatorname{diag}\left(\frac{\sigma_{1}^{+}+\sigma_{1}^{-}}{2}, \frac{\sigma_{2}^{+}+\sigma_{2}^{-}}{2}, \ldots, \frac{\sigma_{n}^{+}+\sigma_{n}^{-}}{2}\right) .
\end{gathered}
$$

Based on Assumption (H2), system (1) can be rewritten as the following form:

$$
\begin{aligned}
\dot{x}_{i}(t)= & -C x_{i}(t)+D f\left(x_{i}(t)\right)+B f\left(x_{i}(t-\tau)\right)+I(t) \\
& +c \sum_{j=1}^{N} G_{i j} A x_{j}(t-\tau) \\
& +c \sum_{j=1}^{N} G_{i j} A_{\tau} x_{j}(t-\tau)-c G_{i i} A_{\tau}\left[x_{i}(t-\tau)-x_{i}(t)\right], \\
\dot{x}_{i}(t)= & -C x_{i}(t)+D f\left(x_{i}(t)\right)+B f\left(x_{i}(t-\tau(t))\right)+I(t) \\
& +c \sum_{j=1}^{N} G_{i j} A x_{j}(t-\tau(t)) \\
& +c \sum_{j=1}^{N} G_{i j} A_{\tau} x_{j}(t-\tau(t)) \\
& -c G_{i i} A_{\tau}\left[x_{i}(t-\tau(t))-x_{i}(t)\right] .
\end{aligned}
$$

The initial conditions of (6) are given by $x_{i}(s)=$ $\phi_{i}(s) . s \in\left[t_{0}-\tau, t_{0}\right], i=1,2, \ldots, N$, where $\phi_{i}(\cdot)=$ $\left[\phi_{i 1}(\cdot), \phi_{i 2}(\cdot), \ldots, \phi_{i n}(\cdot)\right]^{T} \in C\left(\left[t_{0}-\tau, t_{0}\right], R^{n}\right)$. The initial conditions of (7) are given by $x_{i}(s)=\phi_{i}(s) . s \in\left[t_{0}-h_{2}, t_{0}\right]$, $i=1,2, \ldots, N$, where $\phi_{i}(\cdot)=\left[\phi_{i 1}(\cdot), \phi_{i 2}(\cdot), \ldots, \phi_{i n}(\cdot)\right]^{T} \in$ $C\left(\left[t_{0}-h_{2}, t_{0}\right], R^{n}\right)$, and in order to simplify we set $G_{i i}=-l$.

Remark 1. The constants $\sigma_{i}^{-}, \sigma_{i}^{+}$in Assumption (H3) are allowed to be any value. Then, most of the previous results in similar networks are just special cases of this assumption, which means that the activation functions are more general than those of other works. 
Considering the sign of Kronecker product, models (6) and (7) can be rewritten as

$$
\begin{aligned}
\dot{x}(t)= & -\left(I_{N} \otimes C\right) x(t)+\left(I_{N} \otimes D\right) F(x(t))+\left(I_{N} \otimes B\right) \\
& \times F(x(t-\tau))+I^{\prime}(t)+c\left(G \otimes\left(A+A_{\tau}\right)\right) x(t-\tau) \\
& +c l\left(I_{N} \otimes A_{\tau}\right)[x(t-\tau)-x(t)],
\end{aligned}
$$

$$
\begin{aligned}
\dot{x}(t)= & -\left(I_{N} \otimes C\right) x(t)+\left(I_{N} \otimes D\right) F(x(t)) \\
& +\left(I_{N} \otimes B\right) F(x(t-\tau(t))) \\
& +I^{\prime}(t)+c\left(G \otimes\left(A+A_{\tau}\right)\right) x(t-\tau(t)) \\
& +c l\left(I_{N} \otimes A_{\tau}\right)[x(t-\tau(t))-x(t)],
\end{aligned}
$$

with $x(t)=\left[x_{1}^{T}(t), x_{2}^{T}(t), \ldots, x_{N}^{T}(t)\right]^{T}, F(x(t))=$ $\left[f^{T}\left(x_{1}(t)\right), f^{T}\left(x_{2}(t)\right), \ldots, f^{T}\left(x_{N}(t)\right)\right]^{T}$, and $I^{\prime}(t)=$ $\underbrace{\left[I^{T}(t), I^{T}(t), \ldots, I^{T}(t)\right]^{T}}_{N}$.

Definition 2 (see [14]). Dynamical networks (6) are said to be global asymptotic synchronization, if for any $\varepsilon$, any $\varphi_{i}(s), \varphi_{j}(s) \in C\left(\left[t_{0}-\tau, t_{0}\right], R^{n}\right)(i, j=1,2, \ldots, N)$. There exists $T_{1}>t_{0}$ such that $\left\|x_{i}(t)-x_{j}(t)\right\| \leq \varepsilon$, where $t>T_{1}, i, j=$ $1,2, \ldots, N$, and $\|\cdot\|$ denotes the Euclidean norm.

Definition 3 (see [15]). Dynamical networks (7) are said to be global exponential synchronization, if for any initial conditions $\varphi_{i}(s), \varphi_{j}(s) \in C\left(\left[t_{0}-h_{2}, t_{0}\right], R^{n}\right)(i, j=1,2, \ldots, N)$, there exist $T_{1}>t_{0}$ and $\varepsilon>0$ such that $\left\|x_{i}(t)-x_{j}(t)\right\| \leq M e^{-\varepsilon t}$, in which $t>T_{1}$ and $\|\cdot\|$ denotes the Euclidean norm.

Lemma 4. Let $\otimes$ denote the notation of Kronecker product; then one has the following conclusions:

(1) $(\alpha A) \otimes B=A \otimes(\alpha B)$

(2) $(A+B) \otimes C=A \otimes C+B \otimes C$

(3) $(A \otimes B)(C \otimes D)=(A C) \otimes B D$

Lemma 5 (see [16]). Let $e=(1,1, \ldots, 1)^{T}, E_{N}=e e^{T}, U=$ $N I_{N}-E_{N}, K \in R^{n \times n}, x=\left[x_{1}^{T}, x_{2}^{T}, \ldots, x_{N}^{T}\right]^{T}, y=$ $\left[y_{1}^{T}, y_{2}^{T}, \ldots, y_{N}^{T}\right]^{T}$ with $x_{k}, y_{k} \in R^{n}(k=1,2, \ldots, N)$, and then $x^{T}(U \otimes K) y=\sum_{1 \leq i<j \leq N}^{N}\left(x_{i}-x_{j}\right)^{T} K\left(y_{i}-y_{j}\right)$.

Lemma 6 (see [17]). For any constant matrix $X \in R^{n \times n}$, $X=X^{T} \geq 0$, a scalar functional $h:=h(t) \geq 0$, and a vector function $\dot{x}:[-h, 0] \rightarrow R^{n}$, the following inequality holds $-h \int_{t-h}^{t} \dot{x}(s)^{T} \times \dot{x}(s) d s \leq-[x(t)-x(t-h)]^{T} \times[x(t)-x(t-h)]$.

\section{Main Results}

3.1. Synchronization Condition for Constant Time Delay. In this section, we state and investigate the global asymptotic synchronization for system (6).

Theorem 7. Suppose Assumptions (H1)-(H3) hold, if there exist three symmetric positive definite matrices $P, Q, W$ and three positive diagonal matrices $H, K, V$, such that the LMIs hold for each $i, j(i<j)$ in $(1,2, \ldots, N)$. Consider

$$
\Pi_{i j}=\left[\begin{array}{ccccc}
\alpha & -c N G_{i j} P\left(A+A_{\tau}\right)+c l P A_{\tau}+W & -C^{T} K^{T}-c l A_{\tau}^{T} K^{T} & P D+H \Lambda_{2} & P B \\
* & -Q-W-V \Lambda_{1} & \beta & 0 & V \Lambda_{2} \\
* & * & \tau^{2} W-K-K^{T} & K D & K B \\
* & * & * & -H & 0 \\
* & * & * & * & -V
\end{array}\right]<0,
$$

where $\alpha=-P C-C^{T} P+Q-c l P A_{\tau}-c l A_{\tau}^{T} P-W-H \Lambda_{1}, \beta=$ $-c N G_{i j}\left(A+A_{\tau}\right)^{T} K^{T}+c l A_{\tau}^{T} K^{T}$.

Then system (8) is global asymptotic synchronization.

Proof. Choose a Lyapunov-Krasovskii functional as

$$
\begin{aligned}
V(x(t))= & x^{T}(t)(U \otimes P) x(t)+\int_{t-\tau}^{t} x^{T}(s)(U \otimes Q) x(s) d s \\
& +\int_{-\tau}^{0} \int_{t+\theta}^{t} \tau \dot{x}(s)^{T}(U \otimes W) \dot{x}(s) d s d \theta .
\end{aligned}
$$

Now by directly computing $\dot{V}(x(t))$ along the trajectory of system (8), we have

$$
\begin{aligned}
\dot{V}(x(t))= & 2 x^{T}(t)(U \otimes P) \dot{x}(t)+x^{T}(t)(U \otimes Q) x(t) \\
& -x^{T}(t-\tau)(U \otimes Q) x(t-\tau)
\end{aligned}
$$

$$
\begin{aligned}
& +\tau^{2} \dot{x}^{T}(t)(U \otimes W) \\
& \times \dot{x}(t)-\int_{t-\tau}^{t} \tau \dot{x}(s)^{T}(U \otimes W) \dot{x}(s) d s .
\end{aligned}
$$

In view of Lemma 6, we have

$$
\begin{aligned}
\dot{V}(x(t)) \leq & 2 x^{T}(t)(U \otimes P) \dot{x}(t)+x^{T}(t)(U \otimes Q) x(t) \\
& -x^{T}(t-\tau)(U \otimes Q) x(t-\tau) \\
& +\tau^{2} \dot{x}^{T}(t)(U \otimes W) \dot{x}(t) \\
& -[x(t)-x(t-\tau)]^{T}(U \otimes W)[x(t)-x(t-\tau)] .
\end{aligned}
$$


Noting the facts that $(U \otimes P) I(t)=0$ and $U G=N G$, using Lemmas 4 and 5, we have

$\dot{V}(x(t))$

$$
\begin{aligned}
\leq 2 \sum_{i=1}^{N-1} \sum_{j=i+1}^{N}\left(x_{i}(t)-x_{j}(t)\right)^{T}[ & \left(-P C-c l P A_{\tau}+\frac{1}{2} Q\right) \\
& \times\left(x_{i}(t)-x_{j}(t)\right) \\
& +P D\left(f\left(x_{i}(t)\right)-f\left(x_{j}(t)\right)\right) \\
& +P B\left(f\left(x_{i}(t-\tau)\right)\right. \\
& \left.-f N G_{i j} P\left(A+x_{j}(t-\tau)\right)\right) \\
& \times\left(x_{i}(t-\tau)-x_{j}(t-\tau)\right) \\
& +c l P A_{\tau} \\
& \left.\times\left(x_{i}(t-\tau)-x_{j}(t-\tau)\right)\right]
\end{aligned}
$$

$$
\begin{aligned}
-\sum_{i=1}^{N-1} \sum_{j=i+1}^{N} & \left(x_{i}(t-\tau)-x_{j}(t-\tau)\right)^{T} \\
& \times Q\left(x_{i}(t-\tau)-x_{j}(t-\tau)\right)
\end{aligned}
$$$$
+\tau^{2} \sum_{i=1}^{N-1} \sum_{j=i+1}^{N}\left(\dot{x}_{i}(t)-\dot{x}_{j}(t)\right)^{T} W
$$$$
\times\left(\dot{x}_{i}(t)-\dot{x}_{j}(t)\right)
$$$$
-\sum_{i=1}^{N-1} \sum_{j=i+1}^{N}\left[\left(x_{i}(t)-x_{j}(t)\right)^{T} W\left(x_{i}(t)-x_{j}(t)\right)\right.
$$$$
-2\left(x_{i}(t)-x_{j}(t)\right)^{T} W
$$$$
\times\left(x_{i}(t-\tau)-x_{j}(t-\tau)\right)
$$$$
+\left(x_{i}(t-\tau)-x_{j}(t-\tau)\right)^{T} W
$$$$
\left.\times\left(x_{i}(t-\tau)-x_{j}(t-\tau)\right)\right] \text {. }
$$

On the other hand, it is easy to see from the formulation of (8) that the following equation also holds for any matrices $K \in R^{n \times n}$ :

$$
\begin{aligned}
2 \dot{x}^{T}(t)(U \otimes K)( & -\dot{x}(t)-\left(I_{N} \otimes C\right) x(t) \\
& +\left(I_{N} \otimes D\right) F(x(t)) \\
& +\left(I_{N} \otimes B\right) F(x(t-\tau))+I^{\prime}(t) \\
& \left.+c\left(G \otimes\left(A+A_{\tau}\right)\right) x(t-\tau)\right) \\
+c l\left(I_{N} \otimes A_{\tau}\right) & (x(t-\tau)-x(t))=0 .
\end{aligned}
$$

For convenience, let $x_{i j}(t)=x_{i}(t)-x_{j}(t), \dot{x}_{i j}(t)=\dot{x}_{i}(t)-$ $\dot{x}_{j}(t), f\left(x_{i j}(t)\right)=f\left(x_{i}(t)\right)-f\left(x_{j}(t)\right)$, and $f\left(x_{i j}(t-\tau)\right)=$ $f\left(x_{i}(t-\tau)\right)-f\left(x_{j}(t-\tau)\right)$.

For any $n \times n$ diagonal matrices $H>0, V>0$, and $\Lambda_{1}$, $\Lambda_{2}$ from Assumption (H3), we have

$$
\begin{gathered}
\sum_{1 \leq i<j \leq N}\left\{-\left[x_{i j}^{T}(t) H \Lambda_{1} x_{i j}(t)-2 x_{i j}^{T}(t) H \Lambda_{2} f\left(x_{i j}(t)\right)\right.\right. \\
\left.+f^{T}\left(x_{i j}(t)\right) H f\left(x_{i j}(t)\right)\right] \\
-\left[x_{i j}^{T}(t-\tau) V \Lambda_{1} x_{i j}(t-\tau)\right. \\
-2 x_{i j}^{T}(t-\tau) V \Lambda_{2} f\left(x_{i j}(t-\tau)\right) \\
\left.\left.+f^{T}\left(x_{i j}(t-\tau)\right) V f\left(x_{i j}(t-\tau)\right)\right]\right\} \geq 0 .
\end{gathered}
$$

Adding up (14)-(16) from both sides, we have

$\dot{V}(t)$

$$
\begin{aligned}
& \leq \sum_{i=1}^{N-1} \sum_{j=i+1}^{N}\left\{2 x_{i j}(t)^{T}\left(-P C+\frac{1}{2} Q-c l P A_{\tau}\right) x_{i j}(t)\right. \\
& +2 x_{i j}(t)^{T} \operatorname{PD} f\left(x_{i j}(t)\right) \\
& +2 x_{i j}(t)^{T} \operatorname{PBf}\left(x_{i j}(t-\tau)\right) \\
& +2 x_{i j}(t)^{T}\left(-c N G_{i j} P\left(A+A_{\tau}\right)\right) \\
& \left.\times x_{i j}(t-\tau)+2 x_{i j}(t)^{T}\left(c l P A_{\tau}\right) x_{i j}(t-\tau)\right\} \\
& -\sum_{i=1}^{N-1} \sum_{j=i+1}^{N} x_{i j}(t-\tau)^{T} \mathrm{Q} x_{i j}(t-\tau) \\
& +\sum_{i=1}^{N-1} \sum_{j=i+1}^{N} \tau^{2} \dot{x}_{i j}(t)^{T} W \dot{x}_{i j}(t) \\
& -\sum_{i=1}^{N-1} \sum_{j=i+1}^{N}\left\{x_{i j}(t)^{T} W x_{i j}(t)-2 x_{i j}(t)^{T} W x_{i j}(t-\tau)\right. \\
& \left.+x_{i j}^{T}(t-\tau) W x_{i j}(t-\tau)\right\} \\
& -\sum_{i=1}^{N-1} \sum_{j=i+1}^{N}\left\{2 \dot{x}_{i j}^{T}(t) K \dot{x}_{i j}(t)+2 \dot{x}_{i j}^{T}(t)\left(K C+c l K A_{\tau}\right)\right. \\
& \times x_{i j}(t)-2 \dot{x}_{i j}^{T}(t) K D f\left(x_{i j}(t)\right) \\
& -2 \dot{x}_{i j}^{T}(t) K B f\left(x_{i j}(t-\tau)\right) \\
& +2 \dot{x}_{i j}^{T}(t) c N G_{i j} K\left(A+A_{\tau}\right) x_{i j}(t-\tau) \\
& \left.-2 \dot{x}_{i j}^{T}(t)\left(c l K A_{\tau}\right) x_{i j}(t-\tau)\right\}
\end{aligned}
$$




$$
\begin{aligned}
& -\sum_{i=1}^{N-1} \sum_{j=i+1}^{N}\left\{x_{i j}^{T}(t) H \Lambda_{1} x_{i j}(t)\right. \\
& -2 x_{i j}^{T}(t) H \Lambda_{2} f\left(x_{i j}(t)\right) \\
& +f^{T}\left(x_{i j}(t)\right) H f\left(x_{i j}(t)\right) \\
& +x_{i j}^{T}(t-\tau) V \Lambda_{1} x_{i j}(t-\tau) \\
& -2 x_{i j}^{T}(t-\tau) V \Lambda_{2} f\left(x_{i j}(t-\tau)\right) \\
& \left.\quad+f\left(x_{i j}^{T}(t-\tau)\right) V f\left(x_{i j}(t-\tau)\right)\right\} \\
& =\sum_{i=1}^{N-1} \sum_{j=i+1}^{N} \eta_{i j}^{T}(t) \Pi_{i j} \eta_{i j}(t),
\end{aligned}
$$

where $\Pi_{i j}$ is from (10), and

$$
\eta_{i j}^{T}(t)=\left[x_{i j}^{T}(t) x_{i j}^{T}(t-\tau) \dot{x}_{i j}^{T}(t) f\left(x_{i j}^{T}(t)\right) f\left(x_{i j}^{T}(t-\tau)\right)\right] .
$$

Following Theorem 7, we have $V(t) \leq V(0)$, and then we have $x_{i}(t)-x_{j}(t) \rightarrow 0$ for all $1 \leq i<j \leq N$. Therefore, system (8) is global asymptotic synchronization. Then end the proof.

Corollary 8. Suppose Assumptions (H1)-(H3) hold and B $=0$ in the system (8), if there exist three symmetric positive definite matrices $P, Q, W$ and three positive diagonal matrices $H, K, V$, such that the LMIs holds for each $i, j(i<j)$ in $(1,2, \ldots, N)$. Consider

$$
\Pi_{i j}=\left[\begin{array}{ccccc}
\alpha & -c N G_{i j} P\left(A+A_{\tau}\right)+c l P A_{\tau}+W & -C^{T} K^{T}-c l A_{\tau}^{T} K^{T} & P D+H \Lambda_{2} & 0 \\
* & -Q-W-V \Lambda_{1} & \beta & 0 & V \Lambda_{2} \\
* & * & \tau^{2} W-K-K^{T} & K D & 0 \\
* & * & * & -H & 0 \\
* & * & * & * & -V
\end{array}\right]<0
$$

where $\alpha=-P C-C^{T} P+Q-c l P A_{\tau}-c l A_{\tau}^{T} P-W-H \Lambda_{1}, \beta=$ $-c N G_{i j}\left(A+A_{\tau}\right)^{T} K^{T}+c l A_{\tau}^{T} K^{T}$.

Then system (8) is global asymptotic synchronization. details.

The proof is obvious from Theorem 7 and we omit the

3.2. Time-Varying Delay Synchronization Condition. In this section, by utilizing the improved techniques used in [18], we obtain the following global exponential synchronization criterion for system (7).

Theorem 9. Suppose Assumptions (H1)-(H3) hold, if there exist five symmetric positive definite matrices $P, Q_{i}, R_{i}, Z_{1}$,
$Z_{2}$ for each $i$ in $(1,2,3)$ and four positive diagonal matrices $H, K, W, V$, such that the LMIs holds for each $i, j(i<j)$ in $(1,2, \ldots, N)$. Consider

$$
\begin{aligned}
\Phi_{i j}^{1}= & \Phi_{i j}-\left[\begin{array}{llll}
0_{n, 3 n} & -I_{n} & I_{n} & 0_{n, 3 n}
\end{array}\right]^{T} \\
& \times Z_{2}\left[\begin{array}{llll}
0_{n, 3 n} & -I_{n} & I_{n} & 0_{n, 3 n}
\end{array}\right]<0, \\
\Phi_{i j}^{2}= & \Phi_{i j}-\left[\begin{array}{lllll}
0_{n, 3 n} & I_{n} & 0_{n} & -I_{n} & 0_{n, 2 n}
\end{array}\right]^{T} \\
& \times Z_{2}\left[\begin{array}{lllll}
0_{n, 3 n} & I_{n} & 0_{n} & -I_{n} & 0_{n, 2 n}
\end{array}\right]<0,
\end{aligned}
$$

where

$$
\begin{gathered}
\Phi_{i j}=\left[\begin{array}{cccccccc}
\Omega_{11} & \Omega_{12} & \Omega_{13} & \Omega_{14} & Z_{1} & 0 & 0 & 0 \\
* & \Omega_{22} & \Omega_{23} & \Omega_{24} & 0 & 0 & 0 & 0 \\
* & * & \Omega_{33} & \Omega_{34} & 0 & 0 & 0 & 0 \\
* & * & * & \Omega_{44} & Z_{2} & Z_{2} & 0 & 0 \\
* & * & * & * & \Omega_{55} & 0 & W \Lambda_{2} & 0 \\
* & * & * & * & * & -Q_{2}-Z_{2}-V \Lambda_{1} & 0 & V \Lambda_{2} \\
* & * & * & * & * & * & -R_{1}-W & 0 \\
* & * & * & * & * & * & * & -R_{2}-V
\end{array}\right], \\
\Omega_{11}=-P C-C^{T} P+\sum_{i=1}^{3} Q_{i}-c l P A_{\tau}-c l A_{\tau}^{T} P-Z_{1} \\
\\
+
\end{gathered}
$$




$$
\begin{gathered}
\Omega_{12}=-C^{T}\left(h_{1}^{2} Z_{1}+h_{12}^{2} Z_{2}\right) D+P D-c l A_{\tau}^{T}\left(h_{1}^{2} Z_{1}+h_{12}^{2} Z_{2}\right) D+H \Lambda_{2} \\
\Omega_{13}=-C^{T}\left(h_{1}^{2} Z_{1}+h_{12}^{2} Z_{2}\right) B-c l A_{\tau}^{T}\left(h_{1}^{2} Z_{1}+h_{12}^{2} Z_{2}\right) B+P B \\
\Omega_{14}=C^{T}\left(h_{1}^{2} Z_{1}+h_{12}^{2} Z_{2}\right)\left(c N G_{i j}\left(A+A_{\tau}\right)-c l A_{\tau}\right) \\
-c N G_{i j} P\left(A+A_{\tau}\right)+c l P A_{\tau}-c^{2} l N G_{i j} A_{\tau}^{T}\left(h_{1}^{2} Z_{1}+h_{12}^{2} Z_{2}\right) \\
\times\left(A_{\tau}+A\right)-c^{2} l^{2} A_{\tau}^{T}\left(h_{1}^{2} Z_{1}+h_{12}^{2} Z_{2}\right) A_{\tau}, \\
\Omega_{22}=D^{T}\left(h_{1}^{2} Z_{1}+h_{12}^{2} Z_{2}\right) D+\sum_{i=1}^{3} R_{i}-H \\
\Omega_{23}=D^{T}\left(h_{1}^{2} Z_{1}+h_{12}^{2} Z_{2}\right) B \\
\Omega_{24}=D^{T}\left(h_{1}^{2} Z_{1}+h_{12}^{2} Z_{2}\right)\left(-c N G_{i j}\left(A+A_{\tau}\right)+c l A_{\tau}\right) \\
+c l D^{T}\left(h_{1}^{2} Z_{1}+h_{12}^{2} Z_{2}\right) A_{\tau}, \\
\Omega_{33}=B^{T}\left(h_{1}^{2} Z_{1}+h_{12}^{2} Z_{2}\right) B-(1-\mu) R_{3}-K \\
\Omega_{34}=-c N G_{i j} B^{T}\left(h_{1}^{2} Z_{1}+h_{12}^{2} Z_{2}\right)\left(A+A_{\tau}\right)+K \Lambda_{2} \\
+c l B^{T}\left(h_{1}^{2} Z_{1}+h_{12}^{2} Z_{2}\right) A_{\tau}, \\
\Omega_{55}^{2}=-Z_{i j}^{2}\left(A+A_{\tau}\right)^{T}\left(h_{1}^{2} Z_{1}+h_{12}^{2} Z_{2}\right)\left(A+A_{\tau}\right)-(1-\mu) Q_{3}-2 Z_{2}-K \Lambda_{1} \\
\left.A G_{\tau}\right)^{T}\left(h_{1}^{2} Z_{1}+h_{12}^{2} Z_{2}\right) A_{\tau}+c^{2} l^{2} A_{\tau}^{T}\left(h_{1}^{2} Z_{1}+h_{12}^{2} Z_{2}\right) A_{\tau}
\end{gathered}
$$

Then system (9) is global exponential synchronization.

Proof. We construct the Lyapunov-Krasovskii functional as follows:

$$
\begin{aligned}
V(x(t))= & x^{T}(t)(U \otimes P) x(t) \\
& +\int_{t-\tau(t)}^{t} x^{T}(s)\left(U \otimes Q_{3}\right) x(s) d s \\
& +\sum_{i=1}^{2} \int_{t-h_{i}}^{t} x^{T}(s)\left(U \otimes Q_{i}\right) x(s) d s \\
& +\int_{t-\tau(t)}^{t} f^{T}(x(s))\left(U \otimes R_{3}\right) f(x(s)) d s \\
& +\sum_{i=1}^{2} \int_{t-h_{i}}^{t} f^{T}(x(s))\left(U \otimes R_{i}\right) f(x(s)) d s \\
& +\int_{-h_{1}}^{0} \int_{t+\theta}^{t} h_{1} \dot{x}^{T}(s)\left(U \otimes Z_{1}\right) \dot{x}(s) d s d \theta \\
& +\int_{-h_{2}}^{-h_{1}} \int_{t+\theta}^{t} h_{12} \dot{x}^{T}(s)\left(U \otimes Z_{2}\right) \dot{x}(s) d s d \theta .
\end{aligned}
$$

Calculating the time derivative of $V(x(t))$ along the trajectories of system (9), from Lemmas 4 and 5, we have

$$
\begin{aligned}
\dot{V}(x(t))= & 2 x^{T}(t)(U \otimes P) \dot{x}(t)+\sum_{i=1}^{3} x^{T}(t)\left(U \otimes Q_{i}\right) x(t) \\
& -\sum_{i=1}^{2} x^{T}\left(t-h_{i}\right)\left(U \otimes Q_{i}\right) x\left(t-h_{i}\right) \\
& -(1-\mu) x^{T}(t-\tau(t))\left(U \otimes Q_{3}\right) \\
& \times x(t-\tau(t))+\sum_{i=1}^{3} f^{T}(x(t))\left(U \otimes R_{i}\right) f(x(t)) \\
& -\sum_{i=1}^{2} f^{T}\left(x\left(t-h_{i}\right)\right)\left(U \otimes R_{i}\right) f\left(x\left(t-h_{i}\right)\right) \\
& -(1-\mu) f^{T}(x(t-\tau(t)))\left(U \otimes R_{3}\right) \\
& \times f(x(t-\tau(t))) \\
& +\dot{x}^{T}(t)\left[h_{1}^{2}\left(U \otimes Z_{1}\right)+h_{12}^{2}\left(U \otimes Z_{2}\right)\right] \dot{x}(t)
\end{aligned}
$$




$$
\begin{aligned}
& -\int_{t-h_{1}}^{t} h_{1} \dot{x}^{T}(s)\left(U \otimes Z_{1}\right) \dot{x}(s) d s \\
& -\int_{t-h_{2}}^{t-h_{1}} h_{12} \dot{x}^{T}(s)\left(U \otimes Z_{2}\right) \dot{x}(s) d s .
\end{aligned}
$$

Using Lemma 4, we have

$$
\begin{aligned}
& -\int_{t-h_{1}}^{t} h_{1} \dot{x}^{T}(s)\left(U \otimes Z_{1}\right) \dot{x}(s) d s \\
& \quad \leq-\left(x(t)-x\left(t-h_{1}\right)\right)^{T}\left(U \otimes Z_{1}\right)\left(x(t)-x\left(t-h_{1}\right)\right) .
\end{aligned}
$$

On the other hand, based on the approach in [18], and using $h_{12}=\left[h_{2}-\tau(t)\right]+\left[\tau(t)-h_{1}\right]$, then we have

$$
\begin{aligned}
& -\int_{t-h_{2}}^{t-h_{1}} h_{12} \dot{x}^{T}(s)\left(U \otimes Z_{2}\right) \dot{x}(s) d s \\
& =-\int_{t-h_{2}}^{t-\tau(t)} h_{12} \dot{x}^{T}(s)\left(U \otimes Z_{2}\right) \dot{x}(s) d s \\
& -\int_{t-\tau(t)}^{t-h_{1}} h_{12} \dot{x}^{T}(s)\left(U \otimes Z_{2}\right) \dot{x}(s) d s \\
& =-\int_{t-h_{2}}^{t-\tau(t)}\left(h_{2}-\tau(t)\right) \dot{x}^{T}(s)\left(U \otimes Z_{2}\right) \dot{x}(s) d s \\
& -\int_{t-h_{2}}^{t-\tau(t)}\left(\tau(t)-h_{1}\right) \dot{x}^{T}(s)\left(U \otimes Z_{2}\right) \dot{x}(s) d s \\
& -\int_{t-\tau(t)}^{t-h_{1}}\left(\tau(t)-h_{1}\right) \dot{x}^{T}(s)\left(U \otimes Z_{2}\right) \dot{x}(s) d s \\
& -\int_{t-\tau(t)}^{t-h_{1}}\left(h_{2}-\tau(t)\right) \dot{x}^{T}(s)\left(U \otimes Z_{2}\right) \dot{x}(s) d s .
\end{aligned}
$$

And in order to combine some of the terms in (26), letting $\omega=\left(\tau(t)-h_{1}\right) / h_{2}$, we have

$$
\begin{aligned}
& -\int_{t-h_{2}}^{t-\tau(t)}\left(\tau(t)-h_{1}\right) \dot{x}^{T}(s)\left(U \otimes Z_{2}\right) \dot{x}(s) d s \\
& =-\omega \int_{t-h_{2}}^{t-\tau(t)} h_{12} \dot{x}^{T}(s)\left(U \otimes Z_{2}\right) \dot{x}(s) d s \\
& \leq-\omega \int_{t-h_{2}}^{t-\tau(t)}\left(h_{2}-\tau(t)\right) \dot{x}^{T}(s)\left(U \otimes Z_{2}\right) \dot{x}(s) d s \\
& \quad-\int_{t-\tau(t)}^{t-h_{1}} h_{12} \dot{x}^{T}(s)\left(U \otimes Z_{2}\right) \dot{x}(s) d s \\
& =-(1-\omega) \int_{t-\tau(t)}^{t-h_{1}} h_{12} \dot{x}^{T}(s)\left(U \otimes Z_{2}\right) \dot{x}(s) d s \\
& \leq-(1-\omega) \int_{t-\tau(t)}^{t-h_{1}}\left(\tau(t)-h_{1}\right) \dot{x}^{T}(s)\left(U \otimes Z_{2}\right) \dot{x}(s) d s .
\end{aligned}
$$

For any $n \times n$, diagonal matrices $H>0, K>0, W>0$, and $V>0$, and $\Lambda_{1}, \Lambda_{2}$ from Assumption (H3), the following inequality holds:

$$
\begin{aligned}
\sum_{1 \leq i<j \leq N}\left\{-\left[x_{i j}^{T}(t) H \Lambda_{1} x_{i j}(t)-2 x_{i j}^{T}(t) H \Lambda_{2} f\left(x_{i j}(t)\right)\right.\right. \\
\left.+f^{T}\left(x_{i j}(t)\right) H f\left(x_{i j}(t)\right)\right] \\
-\left[x_{i j}^{T}(t-\tau(t)) K \Lambda_{1} x_{i j}(t-\tau(t))\right. \\
\quad-2 x_{i j}^{T}(t-\tau(t)) K \Lambda_{2} f\left(x_{i j}(t-\tau(t))\right) \\
\left.+f^{T}\left(x_{i j}(t-\tau(t))\right) K f\left(x_{i j}(t-\tau(t))\right)\right] \\
-\left[x_{i j}^{T}\left(t-h_{1}\right) W \Lambda_{1} x_{i j}\left(t-h_{1}\right)\right. \\
\quad-2 x_{i j}^{T}\left(t-h_{1}\right) W \Lambda_{2} f\left(x_{i j}\left(t-h_{1}\right)\right) \\
\left.+f^{T}\left(x_{i j}\left(t-h_{1}\right)\right) W f\left(x_{i j}\left(t-h_{1}\right)\right)\right] \\
-\left[x_{i j}^{T}\left(t-h_{2}\right) V \Lambda_{1} x_{i j}\left(t-h_{2}\right)\right. \\
\quad-2 x_{i j}^{T}\left(t-h_{2}\right) V \Lambda_{2} f\left(x_{i j}\left(t-h_{2}\right)\right) \\
\left.\left.+f^{T}\left(x_{i j}\left(t-h_{2}\right)\right) V f\left(x_{i j}\left(t-h_{2}\right)\right)\right]\right\} \geq 0 .
\end{aligned}
$$

Adding up (24)-(28) from both sides, we have

$$
\begin{aligned}
& \dot{V}(x(t)) \\
& \leq \sum_{1 \leq i<j \leq N} \xi_{i j}^{T}(t) \Phi_{i j} \xi_{i j}(t) \\
&-\omega\left[x(t-\tau(t))-x\left(t-h_{2}\right)\right]^{T} \\
& \times\left(U \otimes Z_{2}\right)\left[x(t-\tau(t))-x\left(t-h_{2}\right)\right] \\
&-(1-\omega)\left[x\left(t-h_{1}\right)-x(t-\tau(t))\right]^{T} \\
& \times\left(U \otimes Z_{2}\right)\left[x\left(t-h_{1}\right)-x(t-\tau(t))\right] \\
&= \sum_{1<i<j \leq N} \xi_{i j}^{T}(t)\left[\Phi_{i j}-\omega I_{1}^{T} Z_{2} I_{1}\right. \\
&=\left.\quad-(1-\omega) I_{2}^{T} Z_{2} I_{2}\right] \xi_{i j}(t) \\
& \sum_{i=1}^{N-1} \sum_{j=i+1}^{N} \xi_{i j}(t)^{T}\left[(1-\omega) \Phi_{i j}^{1}+\omega \Phi_{i j}^{2}\right] \xi_{i j}(t),
\end{aligned}
$$

where $\phi_{i j} \phi_{i j}^{1} \phi_{i j}^{2}$ are given in (20), (21), and (9), respectively, and $I_{1}=\left[\begin{array}{lllll}0_{n, 3 n} & -I_{n} & 0_{n} & -I_{n} & 0_{n, 2 n}\end{array}\right]$ and $I_{2}=\left[\begin{array}{llll}0_{n, 3 n} & -I_{n} & I_{n}\end{array}\right.$ $\left.0_{n, 3 n}\right]$. Consider

$$
\begin{aligned}
\xi_{i j}^{T}(t) & \\
= & {\left[\begin{array}{lllll}
x_{i j}^{T}(t) & f^{T}\left(x_{i j}(t)\right) & f^{T}\left(x_{i j}(t-\tau(t))\right) & x_{i j}^{T}(t-\tau(t)) \\
& x_{i j}^{T}\left(t-h_{1}\right) & x_{i j}^{T}\left(t-h_{2}\right) & f^{T}\left(x_{i j}\left(t-h_{1}\right)\right) & f^{T}\left(x_{i j}\left(t-h_{2}\right)\right)
\end{array}\right] . }
\end{aligned}
$$


Following Theorem 9, we have $(1-\omega) \Phi_{i j}^{1}+\omega \Phi_{i j}^{2}<0$, and there exists a positive constant $M>0$ that satisfied (1 $\omega) \Phi_{i j}^{1}+\omega \Phi_{i j}^{2} \leq-M I<0$, such that

$$
\begin{aligned}
\dot{V}(x(t)) & \leq \sum_{i=1}^{N-1} \sum_{j=i+1}^{N} \xi_{i j}(t)^{T}\left[(1-\omega) \Phi_{i j}^{1}+\omega \Phi_{i j}^{2}\right] \xi_{i j}(t) \\
& \leq-M \sum_{i=1}^{N-1} \sum_{j=i+1}^{N}\left[\left\|x_{i j}(t)\right\|^{2}+\left\|x_{i j}(t-\tau(t))\right\|^{2}\right] .
\end{aligned}
$$

Furthermore, based on the proof in [12], there exist two positive scalars $\beta>0$ and $k>0$, such that

$$
\left\|x_{i j}(t)\right\| \leq \beta \sum_{i=1}^{N-1} \sum_{j=i+1}^{N} \sup _{-2 h_{2} \leq s \leq 0}\left\|\varphi_{i}(s)-\varphi_{j}(s)\right\| \cdot e^{-k t}
$$

for $t \geq T_{1}$. By Definition 3, therefore, system (9) is global exponential synchronization. Then end the proof.

Remark 10. In [10-13], the authors studied the synchronization of an array of linearly coupled networks with constant coupling delay or time-varying coupling delay, and the derivative of the time-varying delay is confined to be less than 1. We remove this restrictiveness and the derivative of the time-varying delay can be any value.

Remark 11. During the estimation, $-\int_{t-h_{2}}^{t-h_{1}} h_{12} \dot{x}^{T}(s)\left(U \otimes Z_{2}\right)$ $\dot{x}(s) d s$ is separated into two parts as follows: $-\int_{t-h_{2}}^{t-h_{1}} h_{12} \dot{x}^{T}(s)\left(U \otimes Z_{2}\right) \dot{x}(s) d s=-\int_{t-h_{2}}^{t-\tau(t)} h_{12} \dot{x}^{T}(s)(U \otimes$ $\left.Z_{2}\right) \dot{x}(s) d s-\int_{t-\tau(t)}^{t-h_{1}} h_{12} \dot{x}^{T}(s)\left(U \otimes Z_{2}\right) \dot{x}(s) d s$, and we estimate each part, respectively, ignoring the direct estimate for $-\int_{t-h_{2}}^{t-h_{1}} h_{12} \dot{x}^{T}(s)\left(U \otimes Z_{2}\right) \dot{x}(s) d s$, and then we have more accurate estimate than those from the reference $[12,13]$.

When $h_{1}=0$ in Assumption (H1), we have the following corollary from Theorem 9.

Corollary 12. Suppose Assumptions (H1)-(H3) hold, if there exist four symmetric positive definite matrices $P, Q_{i}, R_{i}, Z_{2}$ for each $i$ in $(1,2)$ and four positive diagonal matrices $H, K, V$, such that the LMIs holds for each $i, j(i<j)$ in $(1,2, \ldots, N)$. Consider

$$
\begin{gathered}
\bar{\Phi}_{i j}^{1}=\bar{\Phi}_{i j}-\left[\begin{array}{lll}
0_{n, 3 n} & -I_{n} & 0_{n, 2 n}
\end{array}\right]^{T} \\
\times Z_{2}\left[\begin{array}{llll}
0_{n, 3 n} & -I_{n} & 0_{n, 2 n}
\end{array}\right]<0 \\
\bar{\Phi}_{i j}^{2}=\bar{\Phi}_{i j}-\left[\begin{array}{llll}
0_{n, 3 n} & I_{n} & -I_{n} & 0_{n}
\end{array}\right]^{T} \\
\times Z_{2}\left[\begin{array}{lllll}
0_{n, 3 n} & I_{n} & -I_{n} & 0_{n}
\end{array}\right]<0 \\
\bar{\Phi}_{i j}=\left[\begin{array}{cccccc}
\bar{\Omega}_{11} & \bar{\Omega}_{12} & \bar{\Omega}_{13} & \bar{\Omega}_{14} & 0 & 0 \\
* & \bar{\Omega}_{22} & \bar{\Omega}_{23} & \bar{\Omega}_{24} & 0 & 0 \\
* & * & \bar{\Omega}_{33} & \bar{\Omega}_{34} & 0 & 0 \\
* & * & * & \bar{\Omega}_{44} & Z_{2} & 0 \\
* & * & * & * & \bar{\Omega}_{55} & V \Lambda_{2} \\
* & * & * & * & * & -R_{2}-V
\end{array}\right]
\end{gathered}
$$

$$
\begin{gathered}
\bar{\Omega}_{11}=-P C-C^{T} P+\sum_{i=1}^{3} Q_{i}-c l P A_{\tau}-c l A_{\tau}^{T} P-Z_{1} \\
+h_{12}^{2} C^{T} Z_{2}\left(C+c l A_{\tau}\right)+c^{2} l^{2} h_{12}^{2} A_{\tau}^{T} Z_{2} A_{\tau} \\
+c l h_{12}^{2} C^{T} Z_{2} A_{\tau}-H \Lambda_{1} \\
\bar{\Omega}_{12}=-h_{12}^{2} C^{T} Z_{2} D+P D-c l h_{12}^{2} A_{\tau}^{T} Z_{2} D+H \Lambda_{2} \\
\bar{\Omega}_{13}=-h_{12}^{2} C^{T} Z_{2} B-c l h_{12}^{2} A_{\tau}^{T} Z_{2} B+P B \\
\bar{\Omega}_{14}=h_{12}^{2} C^{T} Z_{2}\left(c N G_{i j}\left(A+A_{\tau}\right)-c l A_{\tau}\right) \\
-c N G_{i j} P\left(A+A_{\tau}\right)+c l P A_{\tau} \\
-c^{2} l N h_{12}^{2} G_{i j} A_{\tau}^{T} Z_{2}\left(A_{\tau}+A\right) \\
-c^{2} l^{2} h_{12}^{2} A_{\tau}^{T} Z_{2} A \\
\bar{\Omega}_{44}=c^{2} N h_{12}^{2} G_{i j}^{2}\left(A+A_{\tau}\right)^{T} Z_{2}\left(A+A_{\tau} A_{\tau}^{T} Z_{2} A_{\tau}\right. \\
\bar{\Omega}_{55}=-Z_{1}-Z_{2}-Q_{1}-W \Lambda_{1} \cdot \\
\bar{\Omega}_{22}=h_{12}^{2} D^{T} Z_{2} D+\sum_{i=1}^{3} R_{i}-H \\
\bar{\Omega}_{33}=h_{12}^{2} B^{T} Z_{2} B-(1-\mu) R_{3}-K \\
\bar{\Omega}_{24}=h_{12}^{2} D^{T} Z_{2}\left(-c N G_{i j}\left(A+A_{\tau}\right)+c l A_{\tau}\right) \\
\Omega_{23}^{2} D_{12} h_{12}^{2} D^{T} Z_{2} B
\end{gathered}
$$

Then system (9) is global exponential synchronization. The proof is obvious from Theorem 9 and we omit the details. When $\tau(t)$ in Assumption (H1) is not differentiable or $\mu$ is unknown, we have the following corollary from Theorem 9.

Corollary 13. Suppose Assumptions (H1)-(H3) hold, if there exist four symmetric positive definite matrices $P, Q_{i}, R_{i}, Z_{i}$ for each $i$ in $(1,2)$ and four positive diagonal matrices $H, K, W, V$, such that the LMIs holds for each $i, j(i<j)$ in $(1,2, \ldots, N)$. Consider

$$
\begin{aligned}
\widehat{\Phi}_{i j}^{1}= & \widehat{\Phi}_{i j}-\left[\begin{array}{llll}
0_{n, 3 n} & -I_{n} & I_{n} & 0_{n, 3 n}
\end{array}\right]^{T} \\
& \times Z_{2}\left[\begin{array}{llll}
0_{n, 3 n} & -I_{n} & I_{n} & 0_{n, 3 n}
\end{array}\right]<0, \\
\widehat{\Phi}_{i j}^{2}= & \widehat{\Phi}_{i j}-\left[\begin{array}{lllll}
0_{n, 3 n} & I_{n} & 0_{n} & -I_{n} & 0_{n, 2 n}
\end{array}\right]^{T} \\
& \times Z_{2}\left[\begin{array}{lllll}
0_{n, 3 n} & I_{n} & 0_{n} & -I_{n} & 0_{n, 2 n}
\end{array}\right]<0,
\end{aligned}
$$

where 


$$
\widehat{\Phi}_{i j}=\left[\begin{array}{cccccccc}
\widehat{\Omega}_{11} & \widehat{\Omega}_{12} & \widehat{\Omega}_{13} & \widehat{\Omega}_{14} & Z_{1} & 0 & 0 & 0 \\
* & \widehat{\Omega}_{22} & \widehat{\Omega}_{23} & \widehat{\Omega}_{24} & 0 & 0 & 0 & 0 \\
* & * & \widehat{\Omega}_{33} & \widehat{\Omega}_{34} & 0 & 0 & 0 & 0 \\
* & * & * & \widehat{\Omega}_{44} & Z_{2} & Z_{2} & 0 & 0 \\
* & * & * & * & \widehat{\Omega}_{55} & 0 & W \Lambda_{2} & 0 \\
* & * & * & * & * & -Q_{2}-Z_{2}-V \Lambda_{1} & 0 & V \Lambda_{2} \\
* & * & * & * & * & * & -R_{1}-W & 0 \\
* & * & * & * & * & * & * & -R_{2}-V
\end{array}\right] .
$$

With

$$
\begin{aligned}
& \widehat{\Omega}_{11}=-P C-C^{T} P+\sum_{i=1}^{2} Q_{i}-c l P A_{\tau}-c l A_{\tau}^{T} P-Z_{1} \\
& +C^{T}\left(h_{1}^{2} Z_{1}+h_{12}^{2} Z_{2}\right)\left(C+c l A_{\tau}\right) \\
& +c^{2} l^{2} A_{\tau}^{T}\left(h_{1}^{2} Z_{1}+h_{12}^{2} Z_{2}\right) A_{\tau} \\
& +c l C^{T}\left(h_{1}^{2} Z_{1}+h_{12}^{2} Z_{2}\right) A_{\tau}-H \Lambda_{1} \\
& \widehat{\Omega}_{12}=-C^{T}\left(h_{1}^{2} Z_{1}+h_{12}^{2} Z_{2}\right) D+P D \\
& -c l A_{\tau}^{T}\left(h_{1}^{2} Z_{1}+h_{12}^{2} Z_{2}\right) D+H \Lambda_{2} \\
& \widehat{\Omega}_{13}=-C^{T}\left(h_{1}^{2} Z_{1}+h_{12}^{2} Z_{2}\right) B \\
& -c l A_{\tau}^{T}\left(h_{1}^{2} Z_{1}+h_{12}^{2} Z_{2}\right) B+P B \\
& \widehat{\Omega}_{14}=C^{T}\left(h_{1}^{2} Z_{1}+h_{12}^{2} Z_{2}\right)\left(c N G_{i j}\left(A+A_{\tau}\right)-c l A_{\tau}\right) \\
& -c N G_{i j} P\left(A+A_{\tau}\right)+c l P A_{\tau} \\
& -c^{2} l N G_{i j} A_{\tau}^{T}\left(h_{1}^{2} Z_{1}+h_{12}^{2} Z_{2}\right)\left(A_{\tau}+A\right) \\
& -c^{2} l^{2} A_{\tau}^{T}\left(h_{1}^{2} Z_{1}+h_{12}^{2} Z_{2}\right) A_{\tau} \\
& \widehat{\Omega}_{22}=D^{T}\left(h_{1}^{2} Z_{1}+h_{12}^{2} Z_{2}\right) D+\sum_{i=1}^{2} R_{i}-H \\
& \widehat{\Omega}_{23}=D^{T}\left(h_{1}^{2} Z_{1}+h_{12}^{2} Z_{2}\right) B \\
& \widehat{\Omega}_{24}=D^{T}\left(h_{1}^{2} Z_{1}+h_{12}^{2} Z_{2}\right)\left(-c N G_{i j}\left(A+A_{\tau}\right)+c l A_{\tau}\right) \\
& +c l D^{T}\left(h_{1}^{2} Z_{1}+h_{12}^{2} Z_{2}\right) A_{\tau} \\
& \widehat{\Omega}_{33}=B^{T}\left(h_{1}^{2} Z_{1}+h_{12}^{2} Z_{2}\right) B-K \\
& \widehat{\Omega}_{34}=-c N G_{i j} B^{T}\left(h_{1}^{2} Z_{1}+h_{12}^{2} Z_{2}\right)\left(A+A_{\tau}\right)+K \Lambda_{2} \\
& +c l B^{T}\left(h_{1}^{2} Z_{1}+h_{12}^{2} Z_{2}\right) A_{\tau} \\
& \widehat{\Omega}_{44}=c^{2} N G_{i j}^{2}\left(A+A_{\tau}\right)^{T}\left(h_{1}^{2} Z_{1}+h_{12}^{2} Z_{2}\right)\left(A+A_{\tau}\right) \\
& -2 Z_{2}-K \Lambda_{1}
\end{aligned}
$$

$$
\begin{gathered}
-c^{2} l N G_{i j}\left(A+A_{\tau}\right)^{T}\left(h_{1}^{2} Z_{1}+h_{12}^{2} Z_{2}\right) A_{\tau} \\
+c^{2} l^{2} A_{\tau}^{T}\left(h_{1}^{2} Z_{1}+h_{12}^{2} Z_{2}\right) A_{\tau} \\
\widehat{\Omega}_{55}=-Z_{1}-Z_{2}-Q_{1}-W \Lambda_{1}
\end{gathered}
$$

then system (9) is global exponential synchronization.

Remark 14. Theorem 9 can be applied to both slow and fast time-varying delays only if $\mu$ is known. But when $\tau(t)$ is not differentiable or $\mu$ is unknown, Theorem 9 fails to work; however, Corollary 13 can check the synchronization of system (9) instead.

\section{Numerical Examples}

In this section, two examples are provided to illustrate the effectiveness of the conclusion.

Example 1. Consider a lower-dimensional network model with 5 nodes, where each node is a simple three-dimensional stable linear system. To simplify, we assume that $C=$ $\operatorname{diag}(1,2,3), D=B=A_{\tau}=0, I(t)=0$, and the innercoupling matrix is $A=\operatorname{diag}(1,1,1)$, and the outer-coupling matrix is defined as

$$
G=\left[\begin{array}{ccccc}
-2 & 1 & 0 & 0 & 1 \\
1 & -3 & 1 & 1 & 0 \\
0 & 1 & -2 & 1 & 0 \\
0 & 1 & 1 & -3 & 1 \\
1 & 0 & 0 & 1 & -2
\end{array}\right]
$$

We compare the admissible delay upper bounds for $\tau$ and the number of variables with those from other references; it is clear from Table 1 that the delay is a little larger and the number of variables is less than those from $[10,12]$.

Example 2. We consider the following DNNs:

$$
\dot{x}_{i}(t)=-C x_{i}(t)+D f\left(x_{i}(t)\right)+B f\left(x_{i}(t-\tau(t))\right)+I(t) .
$$

Let us see the synchronized states of a chaotic system: Chua's circuit [19]. 
TABLE 1: Admissible delay upper bound $\tau$ for different $c$.

\begin{tabular}{lccc}
\hline Methods & Theorem 1 in [12] & Theorem 1 in [10] & Our result \\
\hline$c=0.3$ & 1.345 & 1.632 & 1.666 \\
$c=0.4$ & 0.950 & 0.998 & 1.000 \\
$c=0.5$ & 0.731 & 0.732 & 0.898 \\
$\begin{array}{l}\text { The number of } \\
\text { variables }\end{array}$ & More than 11 & 7 & 6 \\
\hline
\end{tabular}

The dynamics of Chua's circuit is

$$
\begin{gathered}
\frac{d x_{1}}{d t}=10\left(-x_{1}+x_{2}-f\left(x_{1}\right)\right) \\
\frac{d x_{2}}{d t}=x_{1}-x_{2}+x_{3} \\
\frac{d x_{3}}{d t}=-18 x_{2},
\end{gathered}
$$

where

$$
\begin{array}{r}
f\left(x_{1}\right)=b x_{1}+0.5(d-b)\left(\left|x_{1}+1\right|-\left|x_{1}-1\right|\right), \\
d=-\frac{4}{3}, \quad b=-\frac{3}{4} .
\end{array}
$$

Here we choose Chua's circuit (39) as the uncoupled system. Figure 1 illustrates the chaotic trajectories of system (39). The coupling time-varying delay $\tau(t)=0.03(2+$ $\left.\sin (40 t)+\cos ^{2}(80 t)\right)$ and the coupling strength $c=1$, then we consider dynamic networks consisting of three linearly coupled identical DNNs with couplings as

$$
\begin{aligned}
\dot{x}_{i}(t)= & -C x_{i}(t)+D f\left(x_{i}(t)\right)+B f\left(x_{i}(t-\tau(t))\right)+I(t) \\
& +c \sum_{j=1, j \neq i}^{N} G_{i j} A\left[x_{j}(t-\tau(t))-x_{i}(t-\tau(t))\right] \\
& +c \sum_{j=1, j \neq i}^{N} G_{i j} A_{\tau}\left[x_{j}(t-\tau)-x_{i}(t)\right]
\end{aligned}
$$

for $i=1,2,3$. Let

$$
\begin{gathered}
C=\left[\begin{array}{ccc}
10 & -10 & 0 \\
-1 & 1 & -1 \\
0 & 18 & 0
\end{array}\right], \quad D=\left[\begin{array}{ccc}
-10 & 0 & 0 \\
0 & 0 & 0 \\
0 & 0 & 0
\end{array}\right], \\
G=\left[\begin{array}{ccc}
-4 & 1 & 3 \\
2 & -4 & 2 \\
3 & 1 & -4
\end{array}\right], \quad A=\left[\begin{array}{lll}
1 & 0 & 0 \\
0 & 1 & 0 \\
0 & 0 & 1
\end{array}\right], \\
A_{\tau}=\left[\begin{array}{ccc}
0.5 & 0.1 & 0 \\
0 & 0.5 & 0 \\
0.3 & 0 & 0.5
\end{array}\right],
\end{gathered}
$$

$I(t)=B=0, N=3$, and $l=4$.

By calculating we have $h_{1}=0.06, h_{2}=0.12$, and $\mu=3.6$, and the activation functions $f\left(x_{i}\right)$ satisfy Assumption (H3).

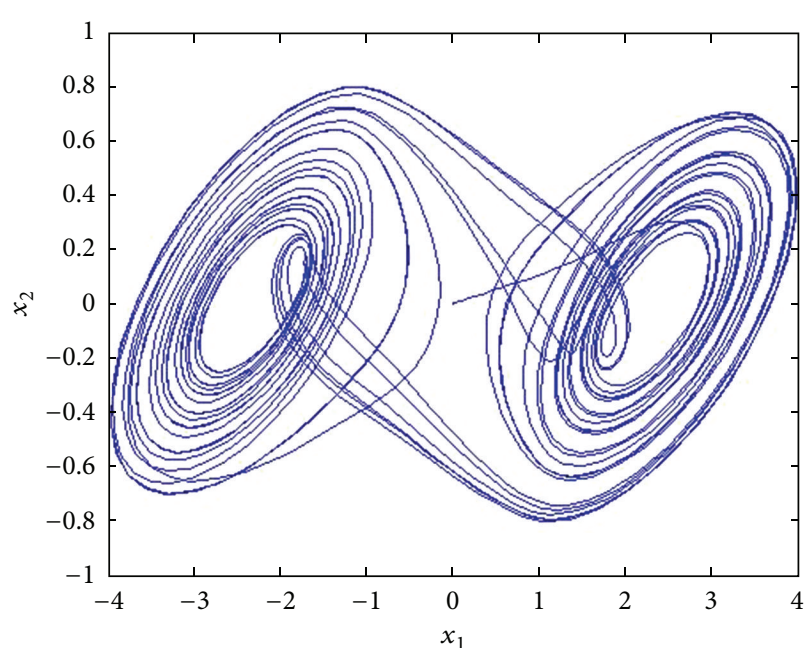

Figure 1: The chaotic trajectories of system (39).

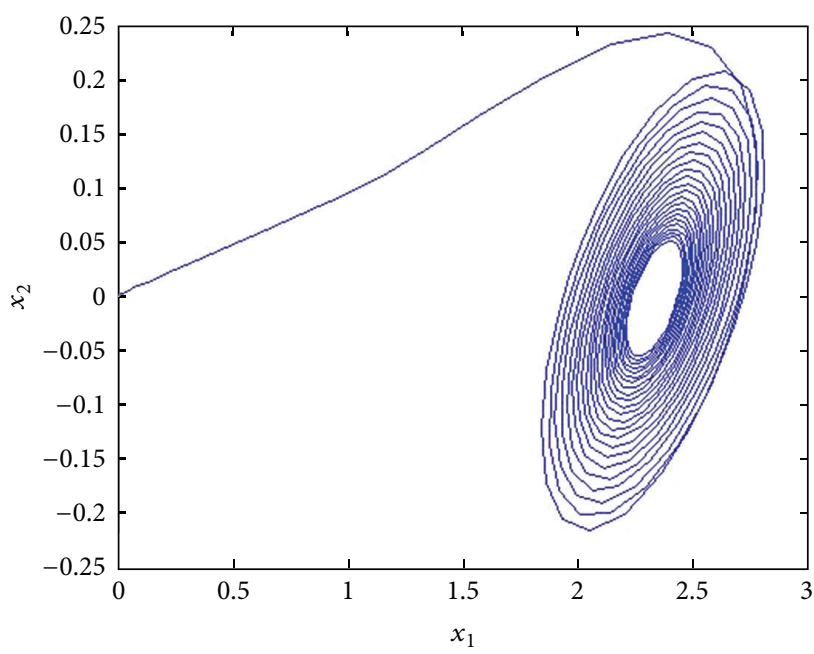

Figure 2: The synchronized state of system (39).

If the system reaches synchronization, we have the following synchronized state equation:

$$
\begin{aligned}
\dot{s}(t)= & -C s(t)+D f(s(t))+B f(s(t-\tau(t))) \\
& +l A_{\tau}(s(t-\tau(t))-s(t)) .
\end{aligned}
$$

By calculating other variables in Theorem 9 using Matlab toolbox, we realize the system synchronization, and the total error is given as error $(t)=\sum_{i=1}^{3} \sqrt{\left[x_{1 i}-x_{2 i}\right]^{2}+\left[x_{2 i}-x_{3 i}\right]^{2}}$. Figures 2 and 3 show the synchronized state of system (39) and the synchronous error, and Figures 4, 5, and 6 show the curves of state variables $x_{i 1}(t), x_{i 2}(t)$, and $x_{i 3}(t)$ with the initial value randomly chosen from $[0,1]$.

\section{Conclusion}

In this paper, global synchronization problem is investigated for general complex networks with coupling delays. Two 


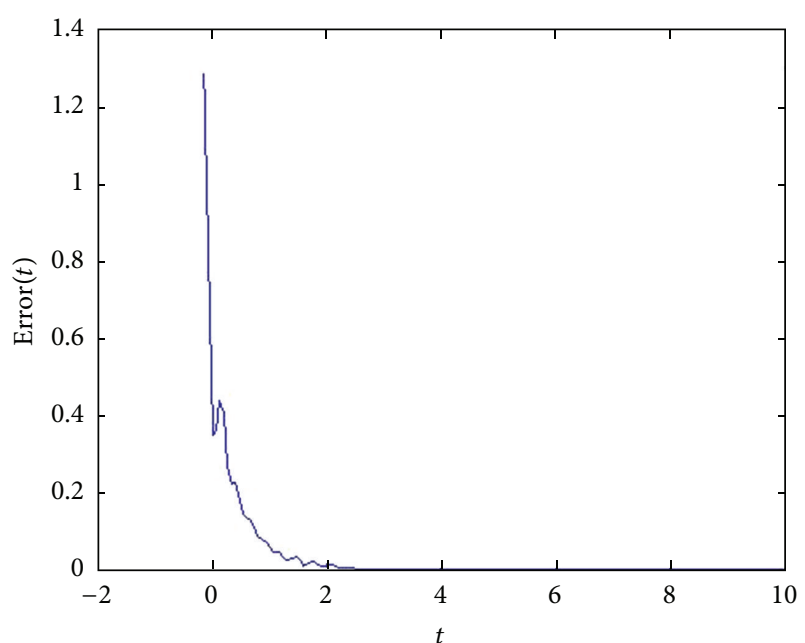

FIGURE 3: The synchronous error of system (39).

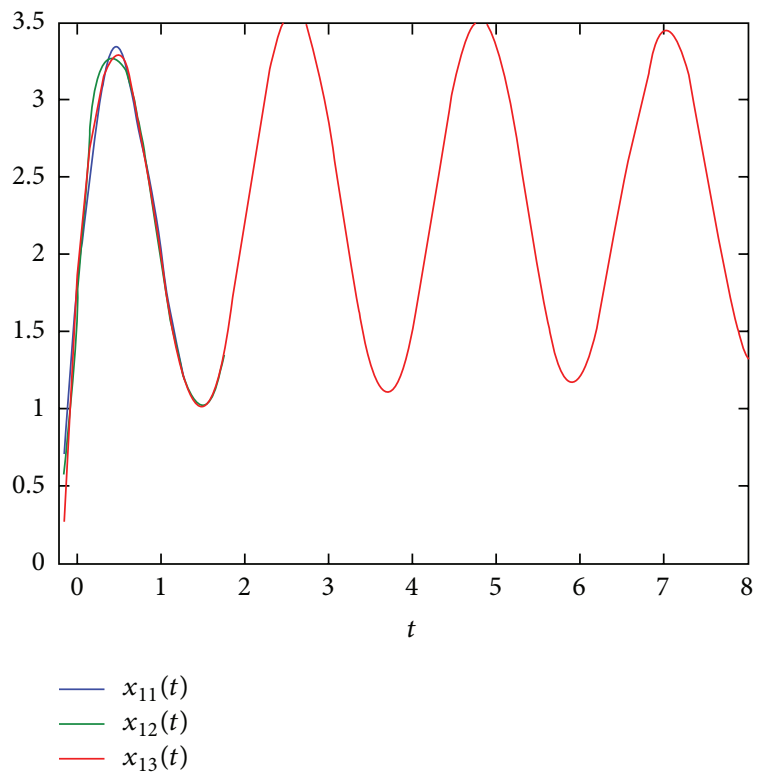

FIGURE 4: The synchronized trajectory of $x_{i 1}(t), i=1,2,3$.

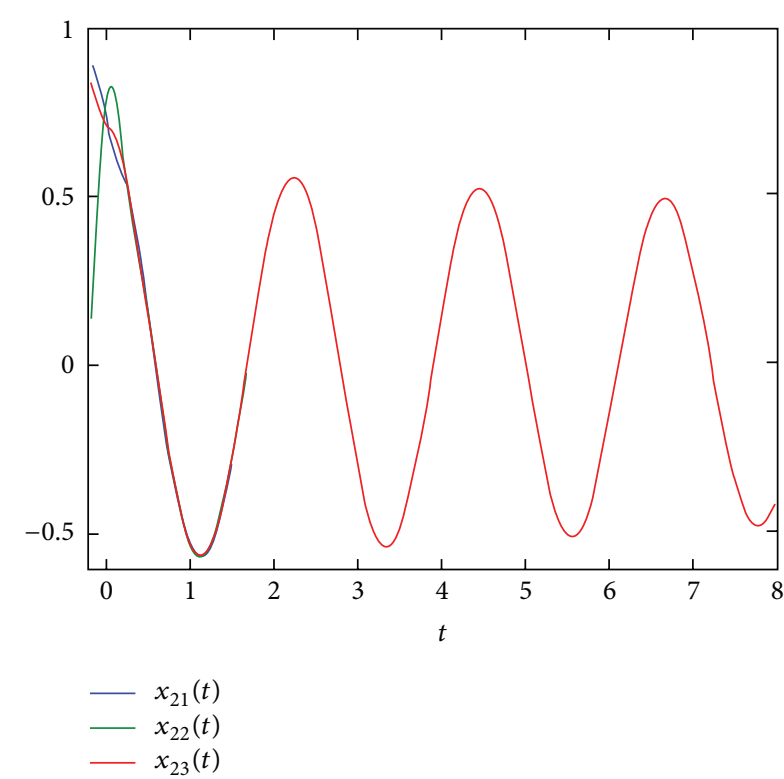

FIGURE 5: The synchronized trajectory of $x_{i 2}(t), i=1,2,3$.

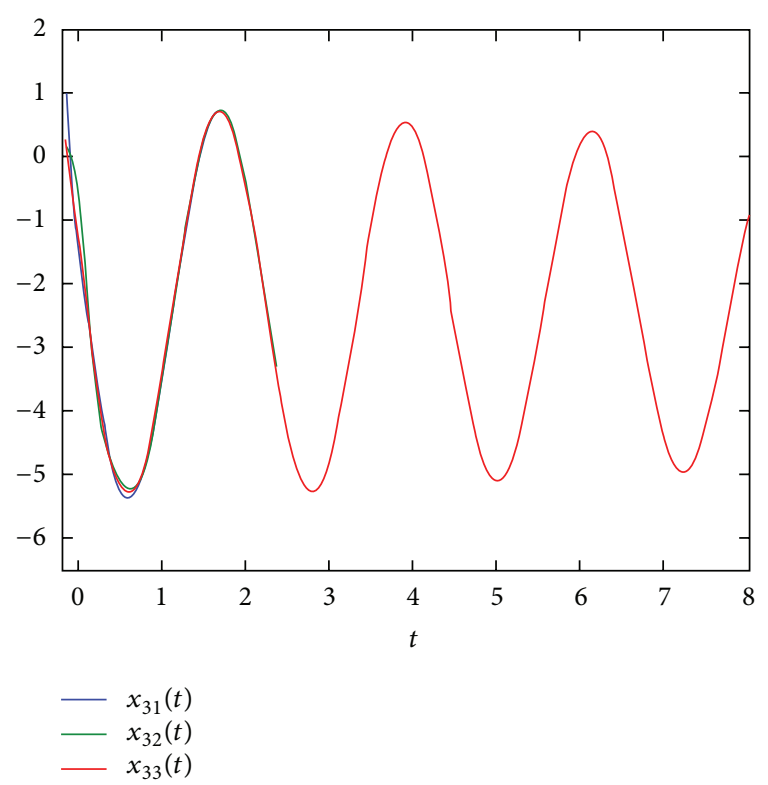

FIgURE 6: The synchronized trajectory of $x_{i 3}(t), i=1,2,3$.

Finally, two examples are given to illustrate the usefulness of the derived methods by the simulation results.

\section{Conflict of Interests}

The authors declare that there is no conflict of interests regarding the publication of the paper.

\section{Acknowledgments}

This work was supported by the National Natural Science Foundation of China (Grant nos. 61070149, 61175115, 61370113, 
and 61272320), the Importation and Development of HighCaliber Talents Project of Beijing Municipal Institutions (Grant no. CIT\&TCD201304035), and Jing-Hua Talents Project of Beijing University of Technology (Grant no. 2014JH-L06).

\section{References}

[1] J. Wang and Z. Xu, "New study on neural networks: the essential order of approximation," Neural Networks, vol. 23, no. 5, pp. 618-624, 2010.

[2] G. Wang, J. Cao, L. Wang, and J. Franklin, "Global dissipativity of stochastic neural networks with time delay," Journal of the Franklin Institute, vol. 346, no. 8, pp. 794-807, 2009.

[3] H. Zhang, Z. Wang, and D. Liu, "Global asymptotic stability of recurrent neural networks with multiple time-varying delays," IEEE Transactions on Neural Networks, vol. 19, no. 5, pp. 855873, 2008.

[4] Z. Yuan, J. Cai, and M. Lin, "Global synchronization in complex networks with adaptive coupling," Mathematical Problems in Engineering, vol. 2010, Article ID 826721, 10 pages, 2010.

[5] J. Lü and G. Chen, "A time-varying complex dynamical network model and its controlled synchronization criteria," IEEE Transactions on Automatic Control, vol. 50, no. 6, pp. 841-846, 2005.

[6] C. Li and G. Chen, "Synchronization in general complex dynamical networks with coupling delays," Physica A, vol. 343, no. 1-4, pp. 263-278, 2004.

[7] J. Zhou and T. Chen, "Synchronization in general complex delayed dynamical networks," IEEE Transactions on Circuits and Systems I, vol. 53, no. 3, pp. 733-744, 2006.

[8] H. Gao, J. Lam, and G. Chen, "New criteria for synchronization stability of general complex dynamical networks with coupling delays," Physics Letters A, vol. 360, no. 2, pp. 263-273, 2006.

[9] W. Guo, F. Austin, and S. Chen, "Global synchronization of nonlinearly coupled complex networks with non-delayed and delayed coupling," Communications in Nonlinear Science and Numerical Simulation, vol. 15, no. 6, pp. 1631-1616, 2010.

[10] D. Gong, H. Zhang, Z. Wang, and B. Huang, "New global synchronization analysis for complex networks with coupling delay based on a useful inequality," Neural Computing and Applications, vol. 22, no. 2, pp. 205-210, 2011.

[11] K. Li, S. Guan, X. Gong, and C.-H. Lai, "Synchronization stability of general complex dynamical networks with timevarying delays," Physics Letters A, vol. 372, no. 48, pp. 7133-7139, 2008.

[12] H. Li and D. Yue, "Synchronization stability of general complex dynamical networks with time-varying delays: a piecewise analysis method," Journal of Computational and Applied Mathematics, vol. 232, no. 2, pp. 149-158, 2009.

[13] Z. Fei, H. Gao, and W. X. Zheng, "New synchronization stability of complex networks with an interval time-varying coupling delay," IEEE Transactions on Circuits and Systems II, vol. 56, no. 6, pp. 499-503, 2009.

[14] W. He and J. Cao, "Global synchronization in arrays of coupled networks with one single time-varying delay coupling," Physics Letters A, vol. 373, no. 31, pp. 2682-2694, 2009.

[15] Q. Song, "Synchronization analysis of coupled connected neural networks with mixed time delays," Neurocomputing, vol. 72, no. 16-18, pp. 3907-3914, 2009.

[16] Y. Wang, Z. Wang, and J. Liang, "A delay fractioning approach to global synchronization of delayed complex networks with stochastic disturbances," Physics Letters A, vol. 372, no. 39, pp. 6066-6073, 2008.

[17] H. Shao, "New delay-dependent stability criteria for systems with interval delay," Automatica, vol. 45, no. 3, pp. 744-749, 2009.

[18] T. Li, T. Wang, A.-G. Song, and S.-M. Fei, "Exponential synchronization for arrays of coupled neural networks with timedelay couplings," International Journal of Control, Automation and Systems, vol. 9, no. 1, pp. 187-196, 2011.

[19] L. O. Chua, “The genesis of Chua's circuit," Archiv für Elektronik und Ubertragung-stechnik, vol. 46, pp. 250-257, 1992. 


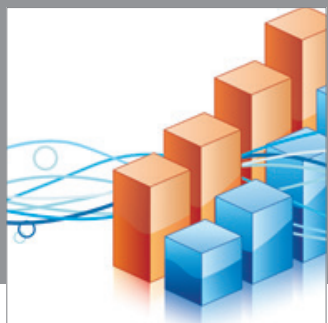

Advances in

Operations Research

mansans

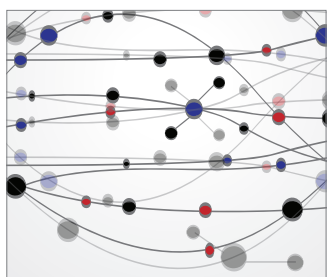

The Scientific World Journal
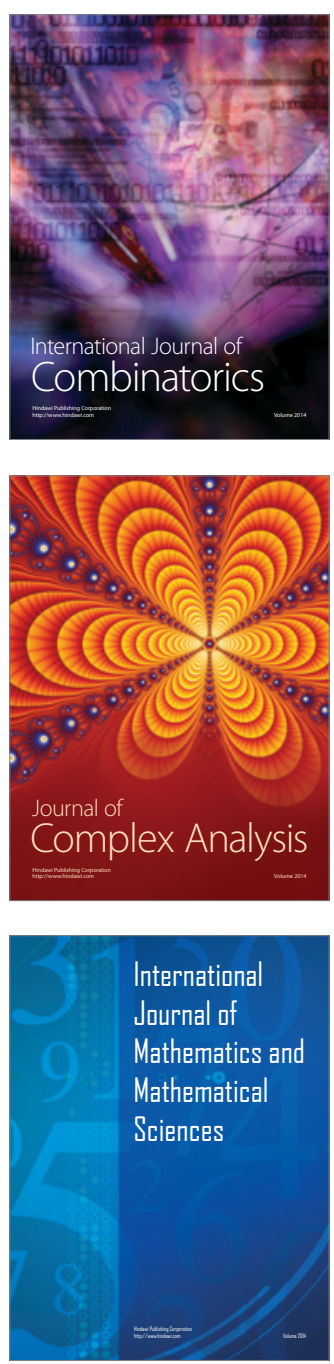
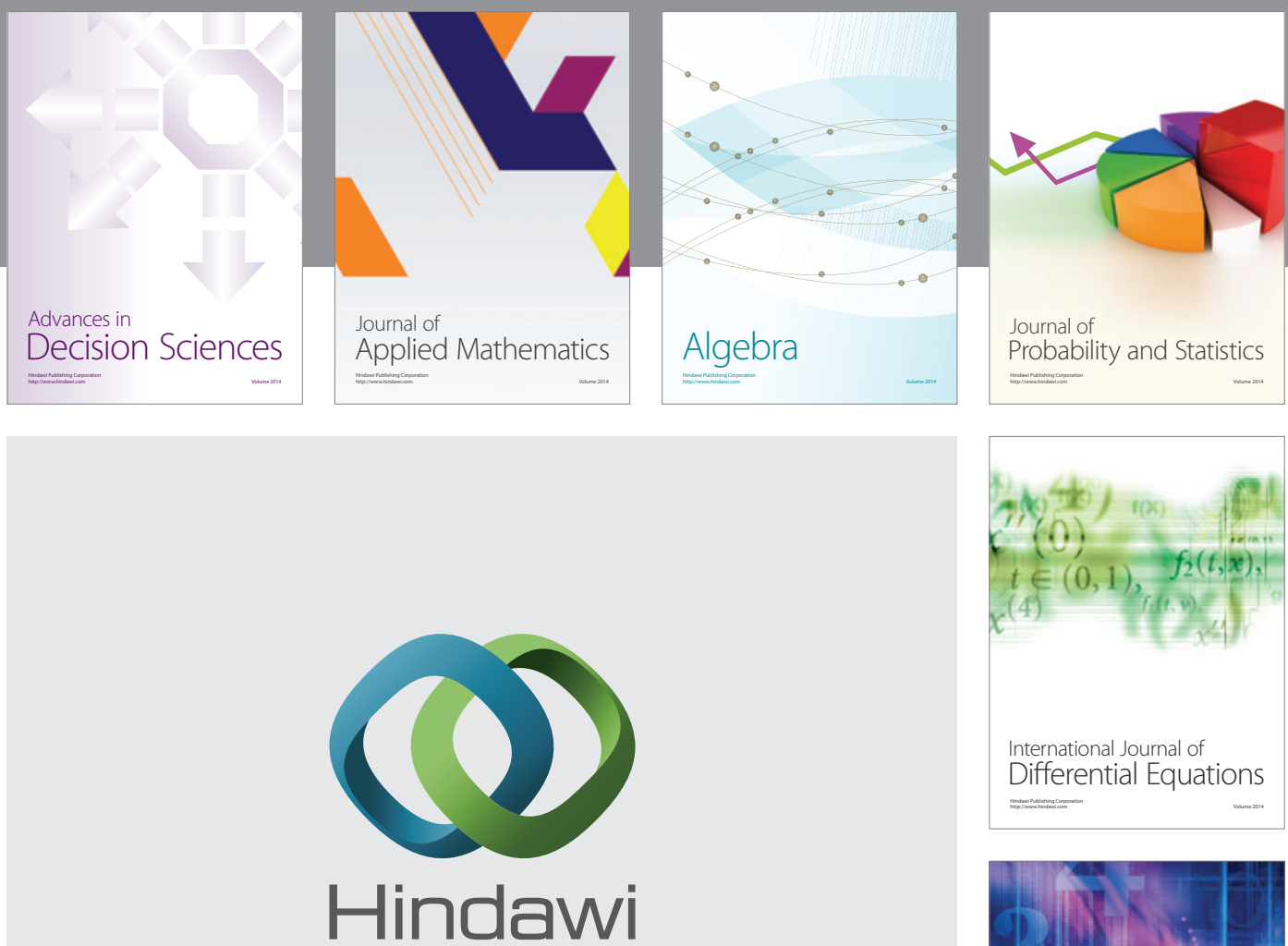

Submit your manuscripts at http://www.hindawi.com
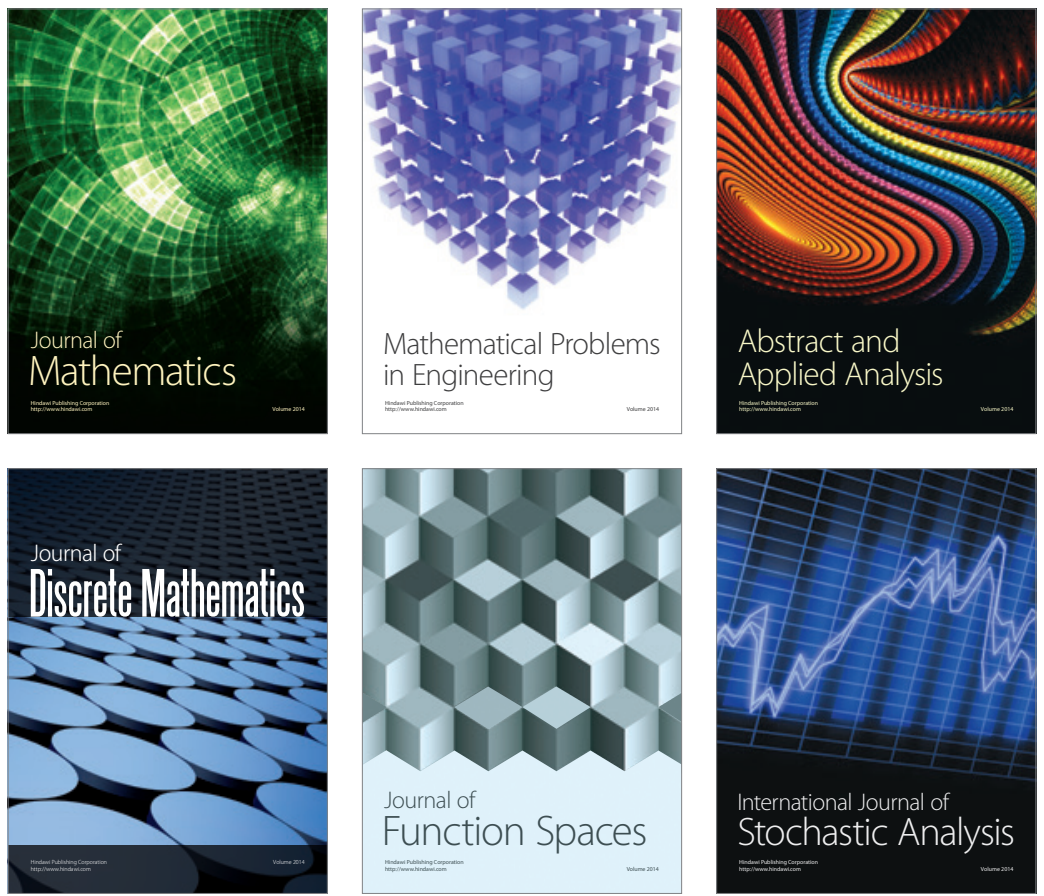

Journal of

Function Spaces

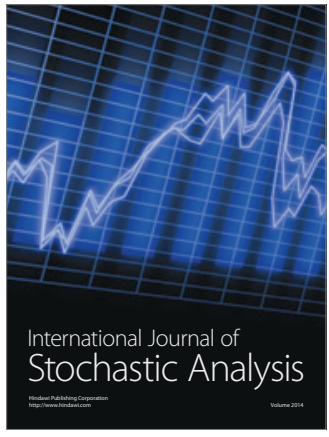

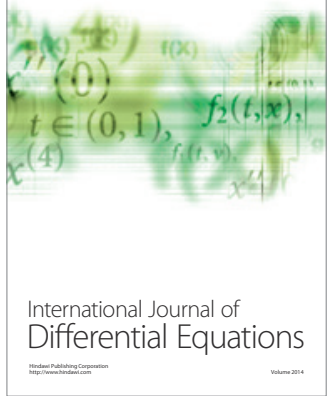
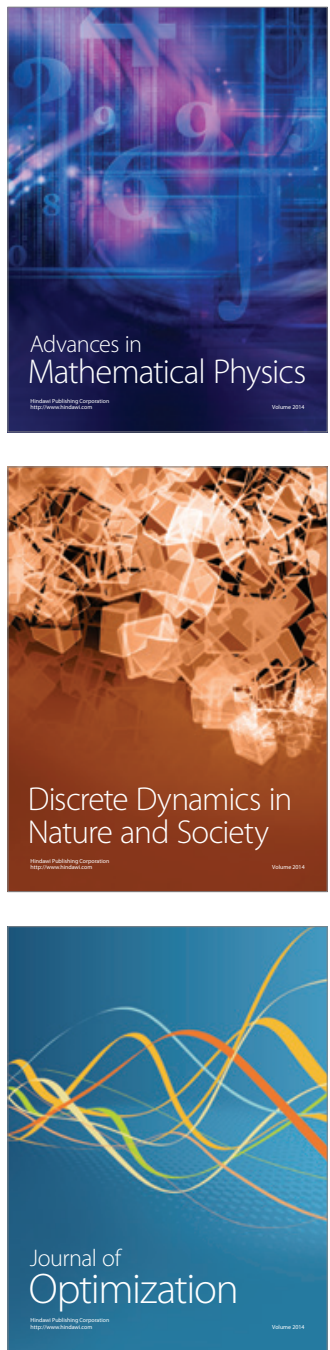